\title{
ECOSSISTEMA E MODELOS DE NEGO?CIOS: UM ESTUDO DE FINTECH UNICO?RNIO POR MEIO DO CASO DE SUCESSO ANT FINANCIAL
}

Thais Helen Sena ${ }^{1}$

Cristiana Fernandes De Muylder ${ }^{2}$

${ }^{1}$ Doutorado em Administração / Faculdade de Ciências Empresariais / FUMEC

${ }^{2}$ UNIVERSIDADE FUMEC 


\section{ECOSSISTEMA E MODELOS DE NEGÓCIOS: UM ESTUDO DE FINTECH UNICÓRNIO POR MEIO DO CASO DE SUCESSO ANT FINANCIAL}

\section{Resumo:}

O surgimento de Startup Financeira ou Fintech, cria a abertura para novos modelos de negócios dentro de um ambiente, até então, tradicional, burocrático e extremamente regulado. A Fintech é considerada uma inovação disruptiva, capaz de causar alteração no mercado de bancos tradicional. Modelo de negócio que promete remodelar o mercado financeiro, com menores custos para as empresas e melhor qualidade de produto e serviço, além de menores custos também para o cliente; o resultado deste "ganha-ganha" é um cenário mais tecnológico, ágil, com mobilidade e mais diversificado. Este artigo apresenta uma visão histórica de Fintech e discute seu ecossistema em relação ao mercado; em seguida, se discute diversos modelos de negócios aplicáveis ao setor. Trata-se de estudo qualitativo bibliográfico com descrição de estudo de caso sobre a análise do conceito de Companhia Unicórnio ilustrada pelos fatores de sucesso da mais valorizada do mundo, a Ant Financial.

Palavras-chave: Modelo de Negócio. Fintech. Unicórnio. Ant Financial.

\section{$1 \quad$ Introdução}

Os bancos tradicionais, estão assistindo o aparecimento de um número crescente de usuários de serviços bancários móveis, e uma das motivações deste comportamento se dá por meio do aumento do número de usuários de celulares. Os benefícios são inúmeros, por exemplo, os usuários não precisam se preocupar em enfrentar filas; e assim, os bancos podem diminuir a equipe nessas agências, transferindo recursos para plataformas de tecnologia da informação que podem atender um número maior de usuários a um custo menor (AHVENAINEN, 2018). Diversos fatos, inclusive os citados, influenciaram o surgimento de Fintechs, que são empresas que apresentam inovações aos mercados financeiros por meio do uso intenso de tecnologia, com potencial para criar novo modelo de negócio; essas empresas atuam por meio de plataformas online e oferecem serviços digitais inovadores relacionados ao setor (BCB, 2019). A Fintech promete aumentar a eficiência e concorrência no mercado de crédito; rapidez e celeridade nas transações; diminuição da burocracia no acesso ao crédito; criação de condições para redução do custo do crédito e inovação (BCB, 2019). O desenvolvimento tecnológico em infraestrutura, big data, análise de dados e dispositivos móveis permitem que as Fintechs possam alterar as finanças tradicionais para empresas com serviços exclusivos, de nicho e personalizados e de acordo com a PwC (2017), 83\% das instituições financeiras acreditam que vários aspectos de seus negócios podem começar a ser operado por empresas de tecnologia. 
O crescimento do investimento em Fintech tem sido considerável, segundo a Deloitte (2018) os investimentos em Fintechs alcançaram US\$34,4 bilhões em todo o mundo em 2017, e quase esse valor nos primeiros seis meses de 2018. No entanto, com a crescente evolução do setor, o terceiro trimestre eleva este número para US\$ 54,4 bilhões, isso incluiu uma rodada de captação de recursos da Ant Financial, rodada avaliada em 14 bilhões de dólares.

A KPMG (2019), relata que o valor do investimento no setor de Fintech em todo o mundo chegou a US\$ 111 bilhões em 2018.

As Fintechs tendem a encontrar lacunas nos bancos tradicionais e preenchê-las com um novo serviço. Elas desafiam os bancos tradicionais, oferecendo novos serviços, como pagamento móvel, empréstimos P2P, gerenciamento de finanças pessoais, moedas virtuais, entre outros (Wubbe, 2018). Segundo Capgemini e Efma (2018), há um impacto das Fintechs em produtos e serviços bancários selecionados, de acordo com executivos bancários do mundo todo em 2018; 66,7\% dos executivos bancários concordam com esta posição.

No entanto, os bancos e as Fintech estão começando a trabalhar juntos, os bancos iniciam programas que incubam Fintechs, 43\% dos bancos fizeram isso em 2015; outra ação vinda dos bancos tradicionais, é criar fundos de risco para financiar Fintech e estabelecer parcerias com eles. Apenas 10\% dos bancos adquiriram sua concorrência e 7\% lançaram suas próprias subsidiárias da Fintech (SKINNER'S, 2015). A análise de Skinner`s (2015) apresenta as seguintes conclusões: (1) a estratégia preferida para a maioria dos bancos é criar programas de inicialização para incubar Fintech com pouco menos da metade delas ou criar fundos de risco para financia-las com $20 \%$ deles escolhendo essa estratégia; (2) como alternativa, um quinto dos bancos analisados adotou parcerias diretas com Fintechs, enquanto alguns bancos adotaram estratégias diferentes adquirindo Fintech ou lançando suas próprias subsidiárias Fintech; (3) aproximadamente 60\% das Fintechs com as quais os bancos se envolvem como parte de qualquer uma das estratégias mencionadas são empresas corporativas Fintech que oferecem soluções de tecnologia aos bancos, enquanto $40 \%$ das Fintech com as quais eles se envolvem oferecem serviços financeiros em suas plataformas diretamente ao varejo e mercado de pequenas empresas; (4) os bancos europeus dominam o envolvimento relacionado à Fintech, com mais de $80 \%$ dos bancos analisados sediados na Europa. Com base na pouca informação disponível, os bancos na Ásia-Pacífico parecem estar adotando uma estratégia mais conservadora e estão trabalhando com algumas das principais empresas tradicionais de TI que possuem seus próprios programas de inovação Fintech. Ainda complementando, quase todos os bancos da lista são grandes bancos de primeira linha com recursos suficientes; (5) deve-se notar que os bancos também estão se engajando cada vez mais com as Fintechs por outros canais, como associações.

Segundo informações do Statista Digital Market Outlook (2018), os EUA lideram o número de empresas Fintech em todo o mundo; especificamente, na Califórnia e em Nova York; por exemplo a SoFi, sediada na Califórnia, é a empresa de fintech mais bem financiada, com mais de US\$ 2 bilhões arrecadados desde 2011. A empresa é apoiada por investidores-chave como SoftBank, Silver Lake Partners, Peter Thiel e outros e foi avaliada em US\$ 4,5 bilhões no primeiro trimestre de 2017. Vários bancos americanos fizeram investimentos em Fintech com a Goldman Sachs, CapitalOne e Citigroup.

Além disso, os bancos também iniciaram centros de inovação focados em várias áreas, como banco móvel, blockchain e criptomoedas, wearables, Internet das Coisas, comércio de 
próxima geração, autenticação, integração biométrica, realidade aumentada e big data (FACTSET, 2017).

Mesmo que os EUA liderem o número de Fintechs, a Ant Financial, é a empresa Fintech mais valorizada do mundo, é a holding dos produtos financeiros da Alibaba. Os principais fatores por trás do sucesso da Ant Financial incluem negócios diversificados, suporte governamental robusto e uma forte presença internacional (CHEN, 2018).

No entanto, apesar de crescente interesse em Fintechs, ainda há falta de consenso sobre sua definição entre estudiosos e profissionais e sobre os fundamentos teóricos deste campo. Além disso, há uma notável falta de trabalhos na academia literatura que trata sistematicamente desse assunto ou que busca mapear de forma a abreviar os levantamentos da literatura existente para apontam para novas atividades de pesquisa e para estruturar conhecimentos nessa área e, assim, poder subsidiar melhor pesquisadores e profissionais (MILIAN et al., 2019).

À luz do necessidade de entender a importância dessa inovação disruptiva, neste artigo, discutiu-se os seguintes tópicos de forma qualitativa e natureza documental: primeiro foi apresentada uma visão histórica de Fintech e discutimos seu ecossistema em relação ao mercado; em seguida, discutiu-se vários modelos de negócios aplicáveis ao setor. Por fím, descreveu-se, com a análise do conceito de Companhia Unicórnio ilustrada pelos fatores de sucesso da mais valorizada do mundo, a Ant Financial.

\section{Aparecimento de Fintech}

O cenário instável dos mercados financeiros desafia o pensamento convencional sobre relacionamentos entre tecnologia, modelos de negócios e serviços financeiros. As empresas Fintech são diferentes das instituições financeiras tradicionais, pelo uso de tecnologia para reimaginar os produtos, serviços e capacidades do setor de serviços financeiros. A revolução da Fintech é caracterizada pela aplicação e síntese de capacidades tecnológicas para reduzir barreiras à entrada e permitir que os recém-chegados se insiram nas cadeias de valor, fornecedores de produtos e serviços inovadores. O resultado dessa reestruturação é que diferentes mecanismos tecnologicamente capacitados para competir e cooperar emergentes estão surgindo em conjunto com novas estratégias para criação de valor (GOZMAN et al., 2018).

A Fintech aparece como um termo recorrente, especialmente na imprensa, como se fosse um fenômeno importante que deveria ser observado pela prática profissionais ligadas ao setor financeiro, tecnologia da informação (TI) e inovação (incubadoras, capital de risco, anjos, entre outros). Derivado da contração das palavras finanças e tecnologia, o termo "Fintech" surgiu na literatura científica em 1972, o vice-presidente da Manufactors Hanover Trust, Abraham Leon Bettinger, trabalhou modelos detalhados sobre como os problemas diários encontrados nessa organização foi analisada e resolvida e formulou os seguintes definição para Fintech: um acrônimo que significa tecnologia financeira, que combina a experiência do banco com a moderna ciência da administração técnicas e o computador (MILIAN et al., 2019).

A teoria que está diretamente relaciona a este evento, é a teoria da inovação disruptiva de Christensen et al., (2015). A Figura 1, mostra o modelo de inovação disruptiva de Christensen 
et al., (2015), e diz que este diagrama contrasta o produto trajetórias de desempenho (a linha vermelha que mostra como produtos ou serviços melhoram com o tempo) com trajetórias de demanda do cliente. Portanto, à medida que as empresas introduzem produtos ou serviços de alta qualidade (linha vermelha superior) para satisfazer as extremo do mercado (onde há maior rentabilidade), eles ultrapassar as necessidades dos clientes. Isso deixa uma abertura para os participantes encontrar pontos de apoio nos segmentos menos rentáveis, onde os tradicionais estão negligenciando.

Figura 1: Modelo de inovação disruptiva

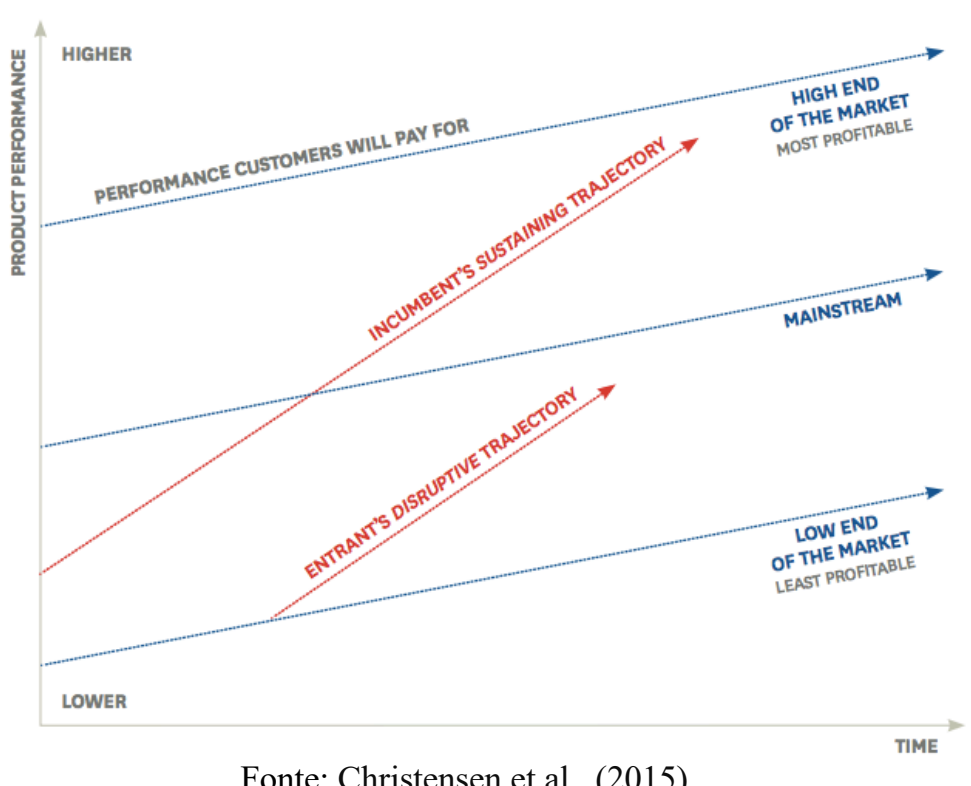

Segundo Milian et al.. (2019) de acordo com a teoria da inovação disruptiva, as Fintechs podem ser classificados em duas categorias: "Fintechs sustentáveis", por prestadores de serviços financeiros estabelecidos que trabalham para proteger suas posições no mercado usando TI por meio de inovações incrementais e "Fintechs disruptivas", que são novas empresas e startups que desafiam as prestadores de serviços oferecendo novos produtos e serviços, e são essas empresas que apresentam novos modelos de negócios que prometem maior flexibilidade, segurança, eficiência e oportunidades do que os serviços financeiros tradicionais.

A taxa de adoção de Fintechs varia de país para país, no entanto a Figura 2 mostra, um domínio do China em algumas áreas. 
Figura 2: Taxa de adoção de Fintech - proporção da população digitalmente ativa usando os seguintes serviços de Fintech, por país (2017).

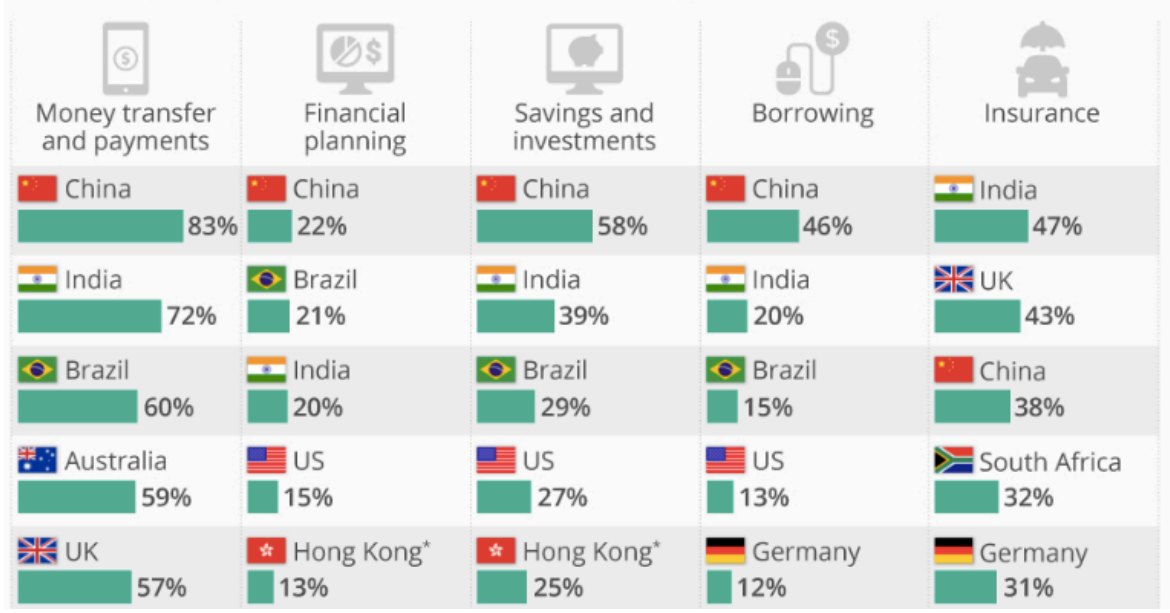

Fonte: EY (2019).

Segundo a EY (2019), a China é a mais avançada no que diz respeito à parcela da população digitalmente ativa usando os serviços de Fintechs. No geral, esse número ficou em $69 \%$ em 2017. Por setor, a China domina em quatro das cinco áreas cobertas. Somente em serviços voltados para seguros, a Índia e o Reino Unido têm uma taxa de adoção mais alta. Em cada setor, os três primeiros são dominados principalmente pela China, Índia e Brasil.

\section{$3 \quad$ Ecossistema Fintech}

Fintech surgem, na busca de novos modelos de negócios, com objetivos de redução de custo, otimização de processos, mobilidade e outros diversos impulsos. Quando se une uma variedade de serviços financeiros, mercados, inovações, participantes do setor, infraestruturas e tecnologias, este ambiente torna-se relativamente complexo (GOZMAN et al., 2018). No entanto, para se entender esta complexidade, é necessário conhecer os atores envolvidos neste ecossistema. Dentro do ecossistema, a inovação é facilitada à medida que novas tecnologias são introduzidas e novas combinações tecnológicas permitem que os modelos de negócios evoluam (GOZMAN et al., 2018).

Lee e Shin (2018), identificaram através de uma revisão da literatura, cinco elementos pertencentes ao ecossistema de Fintech. Schreiber e Vrielink (2019), adotaram os mesmos elementos em seu trabalho, que são representados na Figura 3: 
Figura 3: Ecossistema de Fintech

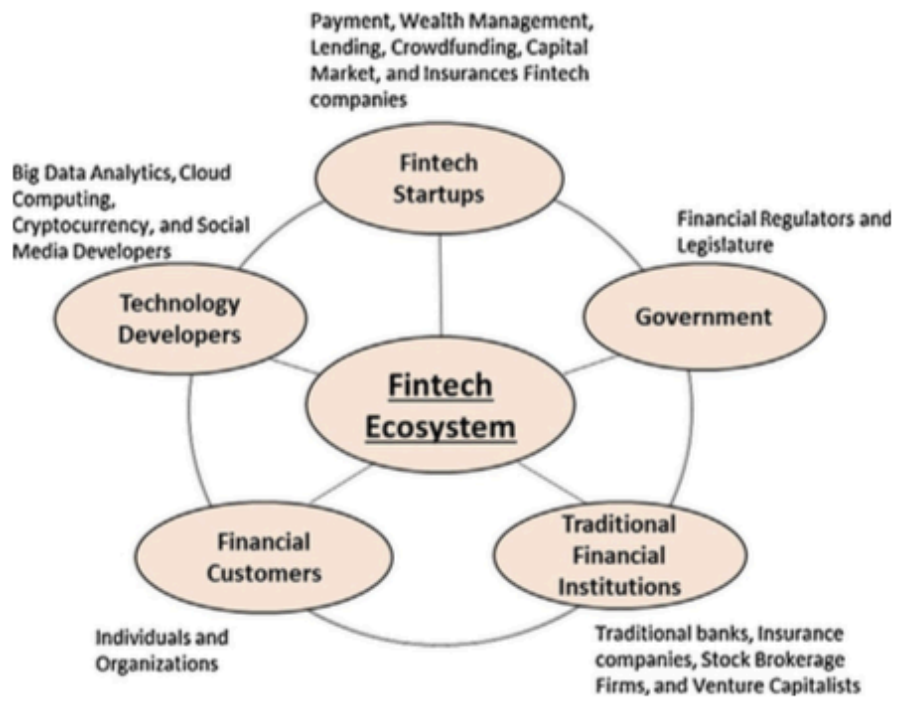

Fonte: Lee e Shin (2018).

Segundo Lee e Shin (2018), dentro deste ecossistema, estão: (1) As Startups Fintech, por exemplo, pagamento, gestão de patrimônio, financiamento, financiamento coletivo, capitalização e empresas de seguros; (2) Desenvolvedores de tecnologia, como análise de big data, computação em nuvem e criptomoeda; (3) Governo, por exemplo, reguladores financeiros; (4) Clientes financeiros, como indivíduos e organizações; e (5) Instituições financeiras tradicionais, como bancos, companhias de seguros e corretora de ações. Suleiman (2018) corrobora com Lee e Shin (2018), mas deixa esse ecossistema aberto, com possibilidade de uma maior amplitude com a possível entrada de novos players, quando diz que devido às mudanças em andamento, surge um novo ecossistema esperado por várias partes interessadas. Esse futuro ecossistema incluirá clientes, Fintech, outros bancos, comunidades de desenvolvedores e players de serviços não financeiros, bem como outros players que podem se juntar no caminho.

\section{$4 \quad$ Modelos de negócio de Fintech}

De acordo com um relatório recente da KPMG (2019), o investimento global das Fintechs mais do que dobrou em 2018. Enquanto novas Fintechs surgiram em subsetores emergentes, áreas altamente maduras como pagamentos viram consolidação. Essas empresas de tecnologia redefiniram as maneiras pelas quais as pessoas armazenam, salvam, emprestar, investir, mover, gastar e proteger dinheiro. A indústria de fintech é um segmento de desenvolvimento dinâmico que combina serviços financeiros e alta tecnologia, que usam tecnologias inovadoras abordagens lógicas aos produtos e serviços financeiros tradicionais; se desenvolvem rapidamente, transformando a cadeia de valor tradicional (ALEKSANDROVNA, 2018). 
Segundo Feng et al. (2001), na literatura os autores se dividem dentro do que chamam de um novo momento da economia; e classificam este momento em quatro segmentos: os visionários, que promoveram a nova economia em livros, por meio de metáfora, como ausência de peso ou excesso, e representou a transformação da nova era que a nova economia traz, por exemplo, Diana Coyle, Jeremy Rifkin e outros; os consultores (e seguidores de escolas de negócios) que posicionaram suas empresas para novas oportunidades econômicas, descrevendo novas regras para o sucesso e prescrevendo o que a administração deve fazer na nova economia, são exemplo, Evans e Wurster da BCG (com HBR) e Meios e Schneider da $\mathrm{PwC}$; os economistas convencionais que se distanciaram da nova economia olhando para os resultados mensuráveis das tecnologias digitais nas taxas de entrada e saída que sugeria cautela sobre a nova economia, por exemplo David Gordon, Paul David e outros; e finalmente os jornalistas Gonzo, que entenderam a nova economia como espuma, são exemplos, Tom Wolfe e Miami Chael Lewis.

Em sua pesquisa, Lee e Shin (2018), identificam na literatura seis modelos de negócios de Fintech, por meio do crescente número de empresas de tecnologia startups: (1) pagamento, (2) gerenciamento de patrimônio, (3) financiamento, (4) empréstimos, (5) mercado de capitais e (6) seguros; que serão trabalhados nesta pesquisa.

\subsection{Modelo de negócio de pagamento}

George Hofheimer, principais executivos de pesquisa e inovação do Instituto de Pesquisa Füene, define o ecossistema de pagamento como "uma rede que move dinheiro entre consumidor, comerciante e contas bancárias usando computadores, software e links de comunicação" Combinando o crescimento de dispositivos mobile com Internet onipresente, conexões e computação em nuvem, se tem um novo modelo de negócios (CREDIT UNION MAGAZINE, 2012. p. 1).

Dentro de um modelo mais amplo, a área líder em gastos bancários em Fintechs, é a área de pagamentos móveis. Segundo Hayashi e Bradford (2014) a escolha de tecnologias afeta significativamente os requisitos para uso de consumidor e comerciante, modelos de negócios e fontes de financiamento; e apresenta as três principais tecnologias que surgiram para pagamentos móveis: (1) NFC (Near Field Communication), que é uma tecnologia de comunicação sem fio baseada em padrões de frequência e permite a troca de dados entre dispositivos compatíveis em proximidade. Isso requer pelo menos um dispositivo para transmitir um sinal e outro para recebê-lo. Os dispositivos NFC podem ser passivos ou ativos; um dispositivo passivo, como um adesivo NFC, contém informações que outros dispositivos podem ler, mas não lê informações em si.; dispositivos ativos, como smartphones, terminais de ponto de venda e outros dispositivos digitais podem ler e enviar informações para outros dispositivos compatíveis. Em termos de segurança, a NFC geralmente estabelece um canal seguro e usa criptografia ao enviar informações confidenciais, como números de cartão de pagamento; (2) QR (código de barras e o código de resposta rápida), que é uma máquina de meios legíveis pelos quais as informações podem ser extraídas.

Barra de códigos lineares, consistem em uma série de linhas pretas verticais e espaços em branco de largura variável, representando números, lidos por um leitor de código de barras para extrair as informações que eles representam. QR Code são formados por padrões de 
quadrados preto e branco normalmente dispostas em uma grade quadrada e podem conter milhares de caracteres numéricos e outros em praticamente qualquer idioma, resolvendo questões da quantidade de informações que eles podem armazenar. (3) Tecnologia em nuvem, utiliza servidores remotos onde os dados podem ser armazenado e elimina limitações de quantos dados podem ser armazenados e a necessidade de fazer investimentos iniciais em software e hardware. A tecnologia utiliza criptografia, utilização de token, e a conexão do dispositivo móvel à Internet para obter credenciais para ativar o pagamento (HAYASHI \& BRADFORD, 2014).

Segundo o Statista Digital Market Outlook (2018), em relação aos tipos de pagamentos digitais, estes incluem pagamentos por produtos e serviços de consumo feitos ao longo do pagamento via Internet e móveis no ponto de venda (PDV) por meio de aplicativos. Há dois tipos de transações: (1) Comércio digital: que são transações de consumidores feitas pela Internet, diretamente relacionadas a compras online de produtos e serviços. As transações online podem ser feitas através de vários pagamentos métodos (cartões de crédito, débito direto, fatura ou fornecedores de pagamento on-line, como PayPal e AliPay). (2) Pagamentos POS móveis: inclui transações no ponto de venda (POS) que são processadas via aplicações para smartphones (chamadas "carteiras móveis"). São exemplos, ApplePay e Samsung Pay. O pagamento nesse caso é feito por uma interação sem contato do aplicativo para smartphone com um terminal de pagamento adequado pertencente ao comerciante. O Statista Digital Market Outlook (2018) apresenta os números de 2017 e faz uma previsão de 2023, afirma que o mercado de pagamentos digitais está crescendo a uma taxa média de crescimento anual de quase $14 \%$ a partir de 2017 até 2023. Os pagamentos de Ponto de Venda (PDV) móveis estão crescendo rapidamente US\$ 357,7 bilhões (12\%) em 2017 para previsão US\$ 2.107,8 bilhões (33\%) até 2023, tudo isso porque empresas Fintech com foco em pagamentos são capazes de adquirir clientes rapidamente a custos mais baixos e são um dos mais rápidos em termos de inovação e adoção de novos recursos de pagamento (LEE e SHIN, 2018).

\subsection{Modelo de negócio de gerenciamento de patrimônio}

O grande número de Fintechs e bancos tradicionais que estão lançando ou comprando participações em consultores de robótica nos últimos anos é um sinal de que o setor de varejo e gerenciamento de patrimônio está se tornando mais automatizado e confiante na inteligência artificial (COMTOIS, 2019). Do ponto de vista do consumidor, existem várias tendências alimentando o crescimento do aconselhamento digital, como: maior transparência nas opções e decisões de investimento; maior acessibilidade por meio de taxas ou mínimos baixos ou inexistentes; experiência aprimorada do cliente via Web e aplicativos móveis; e o uso de fundos negociados em bolsa, ou Exchange Traded Funds (ETFs) para criar diversificado carteiras (KPMG, 2019).

De acordo com o Investment Company Institute (2015), entre 2007 e 2014, houve uma entrada de mais de US\$ 1 trilhão em fundos de índices de ações domésticos, coincidindo com US $\$ 659$ bilhões saída de recursos geridos ativamente por fundos de ações domésticos. Diante dessa tendência, não é surpresa que os investidores estejam interessados em produtos e serviços de consultoria digital (O’KEEFE, 2016). 
Neste segmento, além do Robo-Advisors, que executa serviços de investimento automatizados que permitem que investidores privados alinhem estratégia ou portfólio de investimentos usando recomendações automatizadas, por exemplo, algoritmos, negociação social e parâmetros configuráveis individualmente; ainda há as transferências de dinheiro P2P, que são transferências de dinheiro feitas pela Internet entre indivíduos particulares.

Os pagamentos e remessas internacionais são os segmentos mais relevantes para o mercado Fintech. Este mercado está crescendo de US\$ 288 bilhões em 2017 para uma previsão de US\$ 2678 bilhões em 2023. Robo-Advisors é o maior segmento com 83\% (US \$ 238,8 bilhões) do valor da transação em 2017e previsão de 94\% (US\$ 2.531 bilhões) até 2023 (STATISTA DIGITAL MARKET OUTLOOK, 2018).

O grande diferencial deste modelo de negócio, está em entregar ao cliente de forma on-line, uma assessoria baseada em algoritmos, com personalização. Geralmente estes serviços incluem: rebalanceamento de portfólio baseado em gatilho; coleta de prejuízos fiscais; planejamento simples de aposentadoria com base em objetivos. Além de ter preços mais atrativos e prometer maiores ganhos (BHANOT et al., 2017). Como exemplo, Betterment, Wealthfront, Motivo e Fólio são algumas dessas Fintechs.

\subsection{Modelo de negócio de crowdfunding}

O segmento de mercado de crowfunding refere-se a serviços financeiros digitais para clientes corporativos. Em vista da complexidade do processamento, este mercado normalmente está focado em pequenas e médias empresas e freelancers. No segmento de financiamento alternativo, há dois tipos de transações: (1) crowdinvesting, que é "baseado em ações" e se concentra em Startups que trocam investimentos por ações da empresa e (2) crowdfunding, que é "baseado em recompensa", e são soluções usadas para compensação não monetária, por exemplo, lançamentos de produtos, financiamento de música, arte e cinema (STATISTA DIGITAL MARKET OUTLOOK, 2019).

Neste trabalho, tratou-se do modelo crowfunding, que é um coletivo de abordagem financeira que permite aos indivíduos reunir seus recursos para apoiar projetos favoritos que podem não ter êxito sob um modelo convencional; este modelo de financiamento colaborativo incentiva e prospera na comunidade, como criadores e patrocinadores estabelecem relações estreitas entre si e como o público devolve uma cultura coletiva (BARRETTE, 2011).

Lee e Shin (2018) dizem que o modelo de negócios de crowdfunding envolve três partes: o projeto iniciador ou empresário que precisa de financiamento, o contribuinte que está interessado em apoiar causa ou projeto e a organização moderadora que facilita o engajamento entre as partes tributadores e o iniciador.

A organização moderadora permite que os colaboradores acessem informações sobre as diferentes iniciativas e oportunidades de financiamento organizações para o desenvolvimento de produtos e serviços. Os principais sites de crowdfunding, como IndieGoGo, Kickstart- e o RocketHub servem para dois tipos de projeto: pequenos lançamentos de negócios e grandes projetos. O site de crowdfunding simplifica a organização dos negócios para planejar o projeto, diz às pessoas o objetivo e as vantagens de tributação e permite o acompanhamento do progresso (BARRETTE, 2011). O mercado de financiamento alternativo está crescendo a uma taxa média de crescimento anual superior a 19\% a partir de 2017 até 2023. O 
crowdfunding é o maior segmento, com 57\% (US \$ 3,9 bilhões) da transação do segmento valor em 2017 e 60\% (US \$11,9 bilhões) até 2023 (Statista Digital Market Outlook, 2018).

\subsection{Modelo de negócio de empréstimos}

No segmento de empréstimos, há dois tipos de transações: (1) crowdlending (business): alocação de empréstimos independentes do banco para PMEs; (2) empréstimos no mercado (pessoal): alocação de empréstimos independentes do banco para uso pessoal, ou empréstimo P2P (SCHREIBER; VRIELINK, 2019).

Os empréstimos são feitos através de investidores privados ou institucionais através de plataformas on-line, são exemplos, OnDeck, LendingClub e Prosper (CLAESSENS et al., 2018). O mercado de empréstimos alternativos está crescendo a uma taxa média anual de quase $15 \%$ de 2017 a 2023 . O financiamento coletivo (business) é o maior segmento, com 68\% (US\$ 107,6 bilhões) do valor da transação em 2017 e 79\% (US\$ 288,3 bilhões) até 2023. No entanto, o financiamento médio por empréstimo é comparativamente baixo para empréstimo de mercado (pessoal) em US\$ 2.154 em 2023, em comparação com US\$ 5.699 em Crowdlending (negócios) no mesmo ano (STATISTA DIGITAL MARKET OUTLOOK, 2018). De acordo com Rooney (2019), que apresenta a proporção de saldos de empréstimos pessoais, em todo o mundo de 2010 a 2017, diz que em 2017, as Fintechs detinham 36\% desses saldos, em comparação a menos de $1 \%$ em 2010, enquanto no mesmo ano, os bancos tradicionais detinham 26,4\%. As Fintechs de empréstimos P2P podem oferecer juros baixos e um processo de empréstimo aprimorado para os credores e mutuários. O mercado global $\mathrm{P} 2 \mathrm{P}$ de empréstimos foi avaliado em 3,5 bilhões de dólares em 2013 e a previsão é de chegar em 1 trilhão de dólares em 2025. As principais empresas incluem o Lending Club, Prosper, SoFi, Zopa e RateSetter (THE NUNATAK GROUP, 2018).

A inovação da fintech em empréstimos se manifesta no uso de modelos de crédito alternativos, dados on-line análise de dados para riscos de preços, empréstimos rápidos processos e custos operacionais mais baixos. No entanto, o sucesso ou fracasso desse modelo de negócios, dependente de como as taxas de juros se comportam, algo que as empresas não têm controle algum. Empréstimo P2P e o crowdfunding têm propósitos diferentes: enquanto o principal objetivo do financiamento coletivo é o financiamento para projetos, o principal objetivo dos empréstimos $\mathrm{P} 2 \mathrm{P}$ é consolidação da dívida e refinanciamento de cartão de crédito (LEE; SHIN, 2018)

\subsection{Modelo de negócio de mercado de capitais}

O termo nova economia entrou em uso em meados dos anos 90, quando o poder técnico da conectividade foi demonstrada pela rede de nossos PCs para e-mail e pela Internet, assim como o potencial de criação de riqueza dessas tecnologias foi ilustrado pelo aumento das ações de tecnologia com novos nomes como Cisco e Amazon (FENG et al., 2001).

Novos modelos de negócios da fintech tomam conta de uma espectro de áreas do mercado de capitais, como comércio, câmbio, negociação, gerenciamento de riscos, e pesquisa. Fintechs de negociação permitem que investidores e comerciantes se conectem uns com os outros para discutir e compartilhar conhecimento, fazer pedidos para comprar e vender commodities e 
ações e monitorar riscos em tempo real (LEE; SHIN, 2018). Fintechs oferecendo este serviço são capazes de fazê-lo de custo mais baixo, através de métodos de pagamento muito mais familiar para clientes ou empresas individuais. As Fintechs do mercado de capitais incluem Robinhood, Toro, Magna, Estimize e Xoom (ANIELAK, 2019). Maddock (2017), fazendo uma projeção para 2022, diz que o importante, é que os principais mercados de capitais globais em 2022, provavelmente alcançarão dois objetivos principais: (1) terão usado a tecnologia como uma vantagem competitiva, indo além da simples adoção e redesenho do modelo operacional; e (2) projetam uma organização que assume incerteza, antecipa mudanças, e é capaz de implantar recursos com ritmo e propósito esperado.

\subsection{Modelo de negócio de serviço de seguros}

Nos modelos de negócios de seguros, as Fintechs funcionam para permitir um relacionamento mais direto entre os seguradora e o cliente. Eles usam análise de dados para calcular e combinar o risco, e como o conjunto de ampliados, são oferecidos produtos para atender suas necessidades, por exemplo, carro, vida, saúde, assistência médica ou seguro de causalidade (LEE; SHIN, 2018). A adoção pelo consumidor de empresas e produtos de Fintech cresceu rapidamente em todo o mundo entre 2015 e 2019. A partir de 2019, a adoção de seguros (insurtech) registrou um crescimento significativo de 8\% em 2015 para quase 50\% em 2019, ficando atrás apenas de transferência de dinheiro e serviço de pagamento com $75 \%$ (EY, 2019). Segundo relatório da EY (2019), a China ficou em primeiro lugar com $62 \%$ das pequenas e médias empresas, indicando que usaram pelo menos um serviço da Fintech para seguros, seguida dos EUA com 31\%. Serviços de seguros Fintechs que estão interrompendo o setor de seguros inclui Censio, CoverFox, The Zebra, Sureify Labs e Ladder.

O Statista Digital Market Outlook, 2019, diz que entre 2012 e 2016, havia entre 10 e 15 Fintechs de seguros sendo lançada por mês em todo o mundo, embora há diversos caminhos dentro deste nisso, a direção mais seguida pelas Fintechs é de comparação de preços. No entanto nota-se que não há uma regularidade em lançamentos de Fintech com este modelo de negócio, por no ano de 2016 foram lançadas 208 novas empresas, 109 em 2017 e apenas 12 empresas em 2019 (DELOITTE, 2019).

\section{$5 \quad$ Companhias Unicórnios}

O termo "unicórnio" foi usado pela primeira vez pelo capitalista de risco Aileen Lee em 2 de novembro de 2013, em um artigo do Techcrunch intitulado "Welcome to the unicorn club: learning from bilion dólar Startup", e usou o termo para mostrar quão raro o fenômeno era (COLLOMB; SOK, 2016). Provavelmente existe duas indicações para a comparação de empresas unicórnios: uma é uma empresa criada há mais de dez anos, recebeu investimento em private equity e ainda não foi listado, e a segunda é que a avaliação da empresa é superior a US\$ 1 bilhão (HAN, 2018) e esta é a definição que se enquadra nesta pesquisa.

De Massis et al., (2016) estudou 146 unicórnios identificados pelo The Wall Street Journal, e identificou quatro recursos comuns que ajudam a explicar o sucesso dessas empresas: (1) são pequenas em tamanho, o que permite que as equipes de alta gerência sejam diretamente e profundamente envolvidos na maioria das decisões estratégicas, que são implementadas por 
meio de uma organização plana; o que facilita tomar decisões e colocá-las em prática muito rapidamente; (2) ser liderada por empreendedores em série, os unicórnios são frequentemente fundados e liderados por empreendedores em série, que experimentaram falhas de negócios várias vezes em suas vidas profissionais; (3) financiado por empresas de capital de risco, é improvável que empresas com diferentes estruturas de propriedade, como empresas de capital aberto, serão tão rápidas quanto os unicórnios na criação de suas inovações e trazê-las ao mercado; (4) estreitamente focado, esse foco do tipo laser, implica um compromisso da alta gerência e evita o risco de dispersar a atenção gerencial entre os diferentes desafios. Os unicórnios, se utilizam de plataformas digitais difundidas e redes sociais como canais através do qual eles entram no mercado e alcançam seus clientes-alvo (MARRUGO-SALAS, 2019). Inovações digitais requerem esforços e investimentos menores em marketing e comercialização do que os tradicionais produtos ou serviços, e podem ser difundidos muito mais rapidamente (ACCENTURE, 2019). Em poucas palavras, pode-se resumir que os unicórnios: têm um foco na liderança; seu modelo de negócios é construído em torno de uma única plataforma digital ou software muito rápido e acessível; não precisam investir em construir uma grande força de trabalho e muitos ativos; são apoiados por financiadores motivados por uma venda rápida; e eles estão dispostos a encerrar o negócio ou injetar mais dinheiro em pouco tempo (BRITAIN, 2019).

\subsection{A Ant Financial}

A Ant Financial é a holding dos produtos financeiros da gigante de comércio eletrônico Alibaba. Opera em várias áreas de negócios, incluindo pagamentos digitais: Alipay, finanças empresariais: Ant Micro Loan, empréstimos no mercado: Ant Check Later, gerenciamento de patrimônios: Ant Fortune, banco online: Mybank e seguros e referência de crédito: Sesame Credit (JING, 2018). O Alibaba Group Holding Limited é o principal fornecedor de comércio on-line na China, oferecendo uma amplo gama de serviços de comércio eletrônico B2B, B2C e $\mathrm{C} 2 \mathrm{C}$, bem como pagamentos móveis, além de estar envolvida em serviços de infraestrutura em nuvem, bem como no maior site de vídeo online da China, Youku Tudou. Lançado em 1999 como um portal independente de comércio eletrônico B2B Alibaba.com, o grupo é agora o principal fornecedor de comércio eletrônico na Ásia, pois seu mercado online C2C Taobao e a plataforma de varejo online B2C Tmall também são líderes de mercado em seus respectivos negócios segmentos (STATISTA DIGITAL MARKET OUTLOOK, 2019).

Jing (2018) diz que em vez de competir com bancos e outras empresas financeiras, a Ant vê seu futuro nas vendas poder de computação, sistemas de gerenciamento de riscos sistemas e outras tecnologias. Taxas de tais serviços pode aumentar para $65 \%$ da receita até 2021 , saindo de cerca de 35\% em 2017. Algumas das iniciativas mais recentes da Ant incluem a aplicação Fraude do Shanghai Pudong Development Bank Co. No entanto, todo esse crescimento exponencial não acontece ao acaso, a empresa tem estratégias bem definidas para ocupar o lugar que almeja no mercado.

O Statista Digital Market Outlook (2019) apresenta os principais fatores de sucesso da maior Fintech do mundo:

- Negócios diversificados e big data: a Ant Financial possui um grande número de empresas locais e internacionais. A principal razão para essa participação diversificada é que 
cada uma das empresas é integrada ao imenso ecossistema de comércio eletrônico da Alibaba e obtém benefícios de sua enorme distribuição de rede. Além disso, a riqueza de dados históricos da Alibaba sobre comerciantes e consumidores dá a seus negócios acesso a uma grande base de clientes no momento do lançamento e fornece a eles mais rapidamente conversão de clientes, moderação do risco de crédito e uma infraestrutura on-line de alta escala (STATISTA DIGITAL MARKET OUTLOOK, 2019; GROUP ALIBABA, 2015).

- Investimento do governo: outro motivo que ajuda a impulsionar o sucesso em larga escala da Ant Financial é o envolvimento de instituições financeiras do Estado (STATISTA DIGITAL MARKET OUTLOOK, 2019; GROUP ALIBABA, 2015).

- Instituições como investidores: a China Investment Corporation (CIC), com a riqueza soberana de US\$ 740 bilhões e o fundo nacional de seguridade social do país estão agora entre os maiores acionistas do grupo Alibaba, dando-lhe acesso a uma quantia considerável de dinheiro para financiar seu plano de expansão. Em maio de 2018, a empresa garantiu a maior rodada privada de captação de recursos do mundo por empresa de internet em US\$ 14 bilhões, valorizando cerca de US \$ 150 bilhões (STATISTA DIGITAL MARKET OUTLOOK, 2019; GROUP ALIBABA, 2015).

- $\quad$ Plataforma de nuvem financeira: Em 2010, quando a computação em nuvem estava apenas começando, empresas como Amazon e Google estavam realizando testes e provas de conceitos, o Alibaba montou sua divisão de nuvem chamada Ali Cloud, que ganhou um rápido crescimento, auxiliado pelo fato de que os provedores estrangeiros de nuvem foram impedidos de entrar na China. Em 2013, o Alibaba verticalizou a plataforma com o lançamento do Ali Finance Cloud, que é central para o crescimento de seus negócios de serviços financeiros. A proposição de valor de a plataforma é que pretende ser um balcão único para todas as soluções financeiras, incluindo riscos gerenciamento, depósitos, aplicativos móveis, infraestrutura como serviço, plataforma como serviço, conheça seu cliente (KYC), e outros. A Ant Financial também vende essa solução baseada em nuvem para outros bancos na China (STATISTA DIGITAL MARKET OUTLOOK, 2019; GROUP ALIBABA, 2015).

- $\quad$ Serviços de pagamento offline: Os pagamentos móveis online-offline $(\mathrm{O} 2 \mathrm{O})$ são outra área de crescimento, principalmente devido à sua maior valor da transação comparado aos pagamentos exclusivamente on-line. Com 400 milhões de usuários, a carteira eletrônica Alipay é amplamente utilizada nos pontos de venda chineses, farmácias e restaurantes; onde os usuários podem vincular suas contas bancárias ao serviço e fazer compras na loja, fazendo com que um caixa digitalize um código QR no aplicativo Alipay. Para estimular a expansão internacional, em 2016 a empresa anunciou seus planos de parceria com um milhão de comerciantes off-line na China e no exterior nos próximos três anos para facilitar o uso de Alipay em outros mercados globais. Os ambiciosos planos os colocaram na concorrência com Apple Pay e Samsung Pay, ambos presentes na China, e o pagamento doméstico estabelecido rede UnionPay (STATISTA DIGITAL MARKET OUTLOOK, 2019; QI; XIAO, 2018; GROUP ALIBABA, 2015).

- $\quad$ Expansão internacional: A expansão internacional é um objetivo estratégico novo, mas importante para o estágio atual de crescimento da Ant Financial, e o foco principal está na Ásia. Além de adquirir uma participação significativa na gigante de pagamentos Paytm, a Ant Financial também investiu na empresa de tecnologia de segurança V-Key e parceria com a 
empresa de pagamento tailandesa Ascend. Também possui licenças de carteira digital em países como Tailândia, Indonésia, Filipinas e Vietnã. Na Europa, a Alipay fez parceria com a empresa suíça Global Blue, que permite que os turistas chineses creditem reembolsos de impostos e compras no exterior diretamente para suas contas Alipay (CAPGEMINI; EFMA, 2018).

\section{Conclusão}

Embora exista inúmeros artigos sobre fintech, poucos analisam com profundidade o ecossistema destes novos players. Este artigo se concentrou em estudar os modelos de negócios que envolvem o ecossistema das Fintechs e apresentar como exemplo a maior Fintech em valor financeiro do mundo e como esta Fintech utiliza todos os atores do ecossistema para chegar a esta posição privilegiada.

Verifica-se que os atores do ecossistema das Fintechs não são limitados, por ser um ecossistema que está constantemente em mudança, e com o embasamento da tecnologia, a qualquer momento pode surgir um novo player deste ecossistema. Assim como o ecossistema, os modelos de negócios das Fintechs também são elementos vivos.

Outra vez, com a influência da tecnologia, a qualquer momento pode surgir um modelo que "ninguém pensou". Assim caminha o negócio de Fintech no mundo: altos investimentos, novos atores no ecossistema e novas oportunidades de modelos de negócios. Exemplo disso é a atuação da Fintech mais valiosa do mundo, A Ant Financial.

A Ant Financial, mostra que integrar todos os atores do ecossistema e atuar em vários modelos de negócio, de forma estruturada e estratégica, leva a resultados extraordinários.

No entanto, em uma entrevista da Capgemini e Efma (2018), com 60 executivos bancários entrevistados em todo o mundo, de bancos dos 23 principais mercados, mostrou que dos fatores que mais preocupam no setor bancário global de 2018, são as ofertas bancários das Fintechs, mas principalmente das Bigtechs, ou grandes empresas de tecnologia como Google, Amazon, Facebook, Apple, Microsoft, entre outras. O relatório do Statista Digital Market Outlook (2019) corrobora com Capgemini e Efma (2018) e diz que a última década testemunhou uma movimentação considerável no setor bancário tradicional, especialmente nas áreas de pagamentos, empréstimos, gerenciamento de patrimônio e banco de varejo. Essa mudança não se limitou apenas às Fintechs, grandes empresas de tecnologia e comércio eletrônico, como Google, Amazon, Facebook, Apple e Alibaba, conseguiram alavancar seu enorme alcance e capacidade tecnológica para representar um forte desafio aos concorrentes.

Diante do exposto, surge um novo ator dentro deste mercado foco de análise criteriosa de sua influência no mercado financeiro sugerida para estudo futuro, assim como o estudo de seu modelo de negócio e como se diferencia das Fintechs.

\section{REFERÊNCIAS}

Accenture. (2019). THE WIN-WIN PROPOSITION. Ahvenainen, J. (2018). Two tracks of Asian FinTech in 2018.

Aleksandrovna, Z. O. (2018). ИНСТИТУТЫ FINTECH ACCELERATORS AS THE INSTITUTIONS OF. 
Anielak, K. (2019). Fintech as a Source of Financial Innovations on the Polish Financial

Services Market. Zeszyty Naukowe SGGWw Warszawie - Problemy Rolnictwa

Światowego, 19(34)(1), 162-171. https://doi.org/10.22630/prs.2019.19.1.15

Barrette, B. E. (2011). A communal business model. (152), 32-36.

BCB (2019). Brazilian Central Bank. Retrieved from https://www.bcb.gov.br/estabilidadefinanceira/fintechs

Bhanot, S., Chaudhuri, S., Ghosh, A., Dhamde, S., \& Technology Solutions, C. (2017).

Emerging Trends in Automated Wealth Management Advice. (September). Retrieved from https://www.cognizant.com/whitepapers/emerging-trends-in-automated-wealthmanagement-advice-codex2629.pdf

Britain, H. (2019). Herding Unicorns. CPS.ORG.UK

Capgemini, \& Efma. (2018). Capgemini; Efman - World Retail Banking Report. 1-60.

Retrieved from https://www.capgemini.com/de-de/wp-

content/uploads/sites/5/2018/09/World-Retail-Banking-Report_2018.pdf

Chen, K. (2018). Financial innovation and technology firms: A smart new world with machines. International Symposia in Economic Theory and Econometrics, 25, 279-292. https://doi.org/10.1108/S1571-038620180000025012

Christensen, C. M., Raynor, M., \& McDonald, R. (2015). What is disruptive innovation? Harvard Business Review, 2015(December).

Claessens, S., Zhu, F., Frost, J., \& Turner, G. (2018). Fintech Credit Markets around the World: Size, Drivers and Policy Issues. BIS Quarteley Review, (September), 29-49. Retrieved from https://www.bis.org/publ/qtrpdf/r_qt1809e.pdf

Collomb, A., \& Sok, K. (2016). Blockchain/Distributed Ledger Technology (DLT): What Impact on the Financial Sector? Digiworld Economic Journal, 103(103), 93$111,212,214$.

Comtois, J. (2019). Robo-advisers aren't taking over ... yet. 1-7.

Credit Union Magazine. (2012). Payments Trends Wili Aiter CU Business Medel.

De Massis, A., Frattini, F., \& Quillico, F. (2016). What big companies can learn from the success of the unicorns. Harvard Business Review, (March).

Deloitte. (2019). Accelerating insurance innovation in the age of InsurTech Insurers of the future will need to evolve and transform.

Deloitte (2018). Closing the gap in fintech collaboration: Overcoming obstacles to a symbiotic relationship. 1-16. Retrieved from

https://www2.deloitte.com/global/en/pages/financial-services/articles/gx-the-next-phaseof-fintech-evolution.html

EY. (2019). Gary Hwa EY Global Financial Services Markets Executive Chair and EY AsiaPacific Financial Services Regional Managing Partner.

FactSet. (2017). US M \& A News and Trends. FactSet Flashwire US Monthly, 1-7.

Feng, H., Fourd, J., Johal, S., Haslam, C., \& Willians, K. (2001). A new Business Model? (030), 47.

Gozman, D., Liebenau, J., \& Mangan, J. (2018). The Innovation Mechanisms of Fintech Start-Ups: Insights from SWIFT's Innotribe Competition. Journal of Management Information Systems, 35(1), 145-179. https://doi.org/10.1080/07421222.2018.1440768 
Group Alibaba., Limited, H., \& Baba, N. (2015). Alibaba Group Announces June Quarter 2015 Results. 06(June), 1-23.

Han, R. (2018). Analysis on Risks and Countermeasures of Unicorn Enterprises Based on "Diamond Model." OALib, 05(09), 1-8. https://doi.org/10.4236/oalib.1104867

Hayashi, F., \& Bradford, T. (2014). Mobile Payments: Merchants' Perspectives. Economic Review (01612387), 5-30. Retrieved from http://search.ebscohost.com/login.aspx?direct=true \&db=bth\&AN=97342912\&site=bsilive

Jing, E. (2018). Ant Financial Is Too Big to Ignore. Bloomberg Businessweek, (4574), 25-26. Retrieved from http://libproxy.mit.edu/login?url=http://search.ebscohost.com/login.aspx?direct=true\&db $=$ bth\&AN=130261817\&site $=$ ehost-live\&scope $=$ site

KPMG. (2019). Pulse of Fintech. KPMG-Fintech-Report, (February), 1-85.

Lee, I., \& Shin, Y. J. (2018). Fintech: Ecosystem, business models, investment decisions, and challenges. Business Horizons, 61(1), 35-46. https://doi.org/10.1016/j.bushor.2017.09.003

Maddock, R. (2017). Capital markets. The Cambridge Economic History of Australia, 267286. https://doi.org/10.1017/CHO9781107445222.018

Marrugo-Salas, L. (2018). Unicorns aren't a fairytale, China is their Valley. Online Journal Mundo Asia Pacifico, 7(13), 92-99. https://doi.org/10.17230/map.7.13.6

Milian, E. Z., Spinola, M. de M., \& Carvalho, M. M. d. (2019). Fintechs: A literature review and research agenda. Electronic Commerce Research and Applications, 34(September 2018). https://doi.org/10.1016/j.elerap.2019.100833

O'Keefe, D., Warmund, J., \& Lewis, B. (2016). Robo-advising: catching up or get ahead? Retrieved from https://home.kpmg.com/content/dam/kpmg/pdf/2016/07/Robo-AdvisingCatching-Up-And-Getting-Ahead.pdf

PwC. (2017). FinTech's Growing Influence on Financial Services. 1-12. Retrieved from https://www.pwc.com/jg/en/publications/fintech-growing-influence-financialservices.html.

Qi, B. Y., \& Xiao, J. (2018). Fintech: AI powers financial services to improve people's lives. Communications of the ACM, 61(11), 65-69. https://doi.org/10.1145/3239550

Rooney, K. (2019). Small banks you've never heard of are quietly enabling the tech takeover of the financial industry. Retrieved from https://www.cnbc.com/2019/02/15/small-banksyouve-never-heard-of-quietly-power-the-booming-fintech-industry--.html

Schreiber, T. \& Vrielink, T. (2019). The emergence of FinTech by. (May).

Statista Digital Market Outlook (2018). Fintech Report. Statista.

Statista Digital Market Outlook (2019). Fintech Report. Statista.

Suleiman, A. (2018 Their Interaction with Indonesia 's Evolving Regulatory Governance.

Skinner's, C. (2015). How are banks reacting to Fintech? Retrieved from https://thefinanser.com/2015/02/how-are-banks-reacting-to-

fintech.html/?COLLCC $=2114870184$

The Nunatak Group. (2018). Financial technology. (5), 1-4.

Wubbe, B. Y. E. (2018). The Evolving Fintech / Bank Ecosystem. 34-38. 